Revista Brasileira de Agricultura Irrigada v.10, nº.5, p. 935 - 942, 2016

ISSN 1982-7679 (On-line)

Fortaleza, CE, INOVAGRI - http://www.inovagri.org.br

DOI: $10.7127 /$ rbai.v10n500470

Protocolo 470.16 - 12/08/2016 Aprovado em 26/09/2016

\title{
ESTIMATIVA DA EVAPOTRANSPIRAÇÃO POR DIFERENTES MÉTODOS PARA A CIDADE DE QUIXERAMOBIM, CEARÁ
}

Eldir Bandeira da Silva ${ }^{1}$

\begin{abstract}
RESUMO
O conhecimento da evapotranspiração seja para projeto e/ou manejo de irrigação, assume fundamental importância no volume de água a ser aplicado na irrigação. Com isso, objetivouse com este trabalho avaliar diversos métodos para estimativa da evapotranspiração de referência afim de se obter o melhor ajuste para a região em estudo. O município de Quixeramobim está situado no clima semiárido brasileiro situado na parte central do estado do Ceará, possuindo precipitação pluviométrica média anual é de 707,7 mm e temperatura média anual de $26^{\circ}$ a $28^{\circ} \mathrm{C}$. Foram utilizados dados médios mensais do Instituto Nacional de Meteorologia (INMET) da estação convencional da cidade de Quixeramobim-CE. Os métodos aplicados foram Radiação Solar, Blaney Criddle e Hargreaves-Samani e Penman-Monteith parametrizado pela FAO, sendo este adotado como método padrão aplicado para a região. Os resultados mostraram que o método da Radiação melhor se aplicou a região tendo em vista $o$ coeficiente de determinação de 0,97 . Os demais métodos não mostraram boa correlação, tendo seus coeficientes abaixo de 0,85 . Quando se tem escassez de dados, o método de Radiação é o mais indicado tendo em vista o menor número de entradas e o maior coeficiente de determinação com o método padrão de Penman-Monteith.
\end{abstract}

Palavras-chave: semiárido, agrometeorologia, modelagem.

\section{IN DIFFERENT EVAPOTRANSPIRATION ESTIMATE METHODS FOR QUIXERAMOBIM CITY, CEARÁ}

\begin{abstract}
Knowledge of evapotranspiration is to design and / or management of irrigation, is of fundamental importance in the volume of water to be used in irrigation. With this, objective of this study was to evaluate different methods for estimating reference evapotranspiration in order to obtain the best fit for the region under study. The municipality of Quixeramobim is located in the Brazilian semi-arid climate located in the central part of the state of Ceará, having annual rainfall average is $707.7 \mathrm{~mm}$ and average annual temperature of 26 to $28^{\circ} \mathrm{C}$. Average monthly data were used from the National Institute of Meteorology (INMET) of conventional station in
\end{abstract}

\footnotetext{
${ }^{1}$ Tecnólogo em Irrigação e Drenagem, Mestrando em Engenharia Agrícola na Universidade Federal do Ceará UFC, Fortaleza, Ceará, Brasil. Email: eldir_2005@ hotmail.com
} 
the city of Quixeramobim-CE. The methods applied were Solar Radiation, Blaney Criddle and Hargreaves-Samani and Penman-Monteith parameterized by the FAO, which is adopted as the standard method applied to the region. The results showed that the best method of radiation applied to the region in view of the determination coefficient of 0.97 . The other methods have shown good correlation, with their coefficients below 0.85 . When there is lack of data, the radiation method is the most suitable in view of the smaller number of entries and the highest coefficient of determination with the standard Penman-Monteith.

Keywords: semiarid region, agrometeorology, modeling.

\section{INTRODUÇÃO}

O conhecimento da distribuição espacial e temporal da disponibilidade hídrica de uma região permite estabelecer diretrizes para implementação de políticas de planejamento para o uso racional da água (D'ANGIOLELLA et al., 2005). Em regiões de clima semiárido, onde os recursos hídricos são limitados e seriamente ameaçados pela excessiva exploração, é essencial a estimativa das necessidades hídricas com maior precisão (JABLOUN; SAHLI, 2008). A baixa pluviosidade, altas temperaturas e altas taxas de evaporação podem representar dificuldades para os pequenos agricultores das zonas rurais do Nordeste do Brasil (ANDRADE et al., 2011).

Diante da crescente demanda dos recursos hídricos e os conflitos entre os múltiplos usos, seja na área agrícola que demanda um volume bem maior do que nas atividades urbanas, exige que $o$ planejamento e a gestão da sua utilização ocorram em termos racionais e otimizados. Neste sentido, o correto conhecimento da evapotranspiração das culturas (ETc), seja para projeto e/ou manejo de irrigação, assume fundamental importância no volume de água a ser aplicado na irrigação. Uma das alternativas para se racionalizar o uso da água em projetos agrícolas, é estimar a ETc a partir da evapotranspiração de referência (ETo) (ESTEVES et al., 2010; CARVALHO et al., 2011; TRAJKOVIĆ et al., 2011; MINUZZI et al., 2014).

Existem vários métodos para estimar a ETo, mas o desempenho dos mesmos em diferentes ambientes variam, já que todos eles têm algum conhecimento empírico (SENTELHAS et al.,
2010). No entanto, o método de PenmanMonteith parametrizado pela FAO no seu manual 56, foi estabelecido como um padrão para a determinação da ETo (ALLEN et al., 1998). A estimativa da ETo pela equação de Penman-Monteith FAO 56 só é possível quando um conjunto de dados completos do clima está disponível (SENTELHAS et al., 2010), porém Allen et al. (1998) sugerem que este método também pode ser usado com poucos dados climáticos, estimando os dados em falta.

Em uma abordagem apresentada por CARVALHO et al. (2011) de trabalhos mais recentes sobre desempenho de métodos de estimativa da ETo, concluíram que, quando há disponibilidade de dados para o método de Penman-Monteith FAO- 56 (PM-FAO 56), este deve ser o recomendado como padrão, tendo em vista sua comprovada precisão, independentemente da escala temporal e espacial. Caso contrário, deve-se averiguar um método que esteja de acordo com os dados disponíveis e se é adequado às condições climáticas do local.

Diante disto objetivou-se com este trabalho aplicar diversos métodos para estimativa da evapotranspiração de referência afim de se obter o melhor ajuste para a região em estudo.

\section{MATERIAL E MÉTODOS}

O município de Quixeramobim está situado na parte central do estado, na microrregião geográfica do Sertão de Quixeramobim (Figura 1). O clima é do tipo tropical quente semiárido, enquadrando-se na classificação climática de 
Köppen como BSw'h'. A precipitação $\mathrm{mm}$ e temperatura média anual de $26^{\circ}$ a pluviométrica média anual é de $707,7 \quad 28^{\circ} \mathrm{C}$.

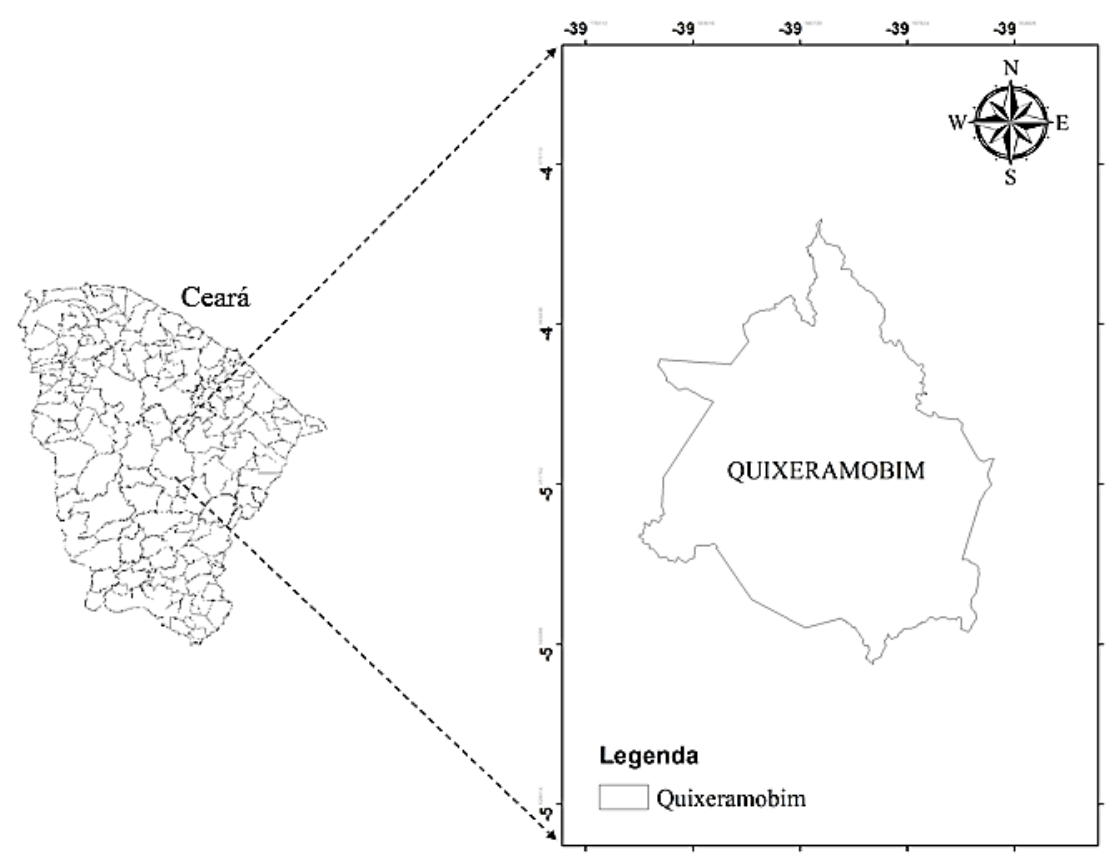

Figura 1 - Localização do município de Quixeramobim, Ceará.

Foram utilizados dados médios mensais do Instituto Nacional de Meteorologia (INMET) da estação convencional da cidade de QuixeramobimCE. Os elementos climáticos estudados foram: umidade relativa do ar, velocidade do vento, temperatura do ar e insolação. As estimativas da evapotranspiração de referência diária (ETo) foram obtidas pelos modelos de Penman-Monteith parametrizado pela FAO, Radiação Solar (DOORENBOS; PRUITT, 1977), Blaney Criddle (BLANEY; CRIDDLE, 1950) e Hargreaves-Samani (HARGREAVES;
SAMANI, 1985). Estes métodos foram selecionados por serem recomendados pelos Boletins 24 e 56 da FAO para a estimativa da evapotranspiração de referência.

A FAO recomenda que os modelos empíricos devem ser comparados com a equação de Penman-Monteith (Equação 1), (ALLEN et al, 1998). Este modelo foi utilizado como padrão no presente estudo por apresentar excelente desempenho na estimativa da ETo em uma grande variedade de condições climáticas para o intervalo de tempo de 24 horas.

$$
\mathrm{ET}_{\mathrm{o}}=\frac{0,408 \Delta\left(\mathrm{R}_{\mathrm{n}}-\mathrm{G}\right)+\gamma \frac{900}{\mathrm{~T}_{\mathrm{m}}+273} \mathrm{u}_{2}\left(\mathrm{e}_{\mathrm{s}}-\mathrm{e}_{\mathrm{a}}\right)}{\Delta+\gamma\left(1+0,34 \cdot \mathrm{u}_{2}\right)}
$$

Em que:

$\mathrm{ET}_{\mathrm{o}}$-evapotranspiração de referência, $\mathrm{mm}$ $\operatorname{dia}^{-1} ; \mathrm{R}_{\mathrm{n}}$-radiação líquida total do gramado, $\mathrm{MJ}$ $\mathrm{m}^{-2} \mathrm{dia}^{-1}$; G-densidade do fluxo de calor no solo, $\mathrm{MJ} \mathrm{m}{ }^{-2} \mathrm{dia}^{-1} ; \mathrm{T}_{\mathrm{m}}$-temperatura média do ar, ${ }^{\circ} \mathrm{C}$; $\mathrm{u}_{2}$-velocidade do vento média diária a $2 \mathrm{~m}$ de altura, $\mathrm{m} \mathrm{s}^{-1}$; es-pressão de saturação de vapor, $\mathrm{kPa}$; ea-pressão parcial de vapor, $\mathrm{kPa} ; \Delta$ - declividade da curva de pressão de vapor no ponto de $\mathrm{Tm}, \quad \mathrm{kPa}^{\circ} \mathrm{C}^{-1} ; \quad \gamma$-coeficiente psicrométrico, $\mathrm{kPa}^{\circ} \mathrm{C}^{-1}$.

As equações padronizadas para o cálculo de todos os parâmetros da equação (1) são apresentadas em Allen et al. (1998).

Com o objetivo de melhor definir os efeitos do clima nas necessidades hídricas das 
culturas, o método de Blaney-Criddle original (BLANEY; CRIDDLE, 1950) foi modificado por Doorenbos e Pruitt (1977) para a obtenção da ETo, considerando os níveis gerais de umidade relativa mínima, velocidade do vento e insolação. $\mathrm{O}$ método de Blaney-Criddle modificado pela FAO é o seguinte:

$$
\begin{aligned}
& E T o_{B C}=a+b \cdot p\left(0.46 T_{\text {med }}+8.13\right) \\
& a=0.0043 U R_{\min }-\frac{n}{N}-1.41 \\
& b=a_{0}+a_{1} U R_{\text {min }}+a_{2} \frac{n}{N}+a_{3} u_{2}+a_{4} U R_{\min }\left(\frac{n}{N}\right)+a_{5} U R_{\min } u_{2}
\end{aligned}
$$

Em que: $\mathrm{UR}_{\min }$ é a umidade relativa mínima mensal, $\mathrm{n} / \mathrm{N}$ é a razão da insolação média mensal, $\mathrm{u}_{2}$ é a velocidade média do vento a 2 metros de altura $\left(\mathrm{m} \cdot \mathrm{s}^{-1}\right), \mathrm{a}_{0}=0,81917, \mathrm{a}_{1}=$ $0,0040922, a_{2}=1,0705, a_{3}=0,065649, a_{4}=-$ 0,0059684 e $\mathrm{a}_{5}=-0,0005967$.

O método da radiação é uma adaptação feita por Doorenbos e Pruitt (1977) e Doorenbos e Kassam (1994) para o método de Makkink, em que os coeficientes da equação de Makking foram substituídos por um parâmetro $\mathrm{r}$, em função da umidade relativa do ar e da velocidade do vento (PEREIRA et al. 1997). A estimativa da evapotranspiração é dada pela seguinte equação.

$$
\begin{aligned}
& E T o_{R S}=a+b \cdot\left(W \cdot R_{s}\right) \\
& W=\frac{\Delta}{\Delta+\gamma}
\end{aligned}
$$

$$
R_{s}=\left(0.25+0.5 \frac{n}{N}\right) R a
$$

Em que: a e b são as equações 3 e 4, respectivamente; $\Delta$ é a inclinação da curva de pressão de saturação e vapor e temperatura do ar; Y é a constante psicrométrica; Rs: radiação solar global; $\mathrm{R}_{\mathrm{a}}$-radiação solar no topo da atmosfera, $\mathrm{MJ} \mathrm{m}^{-2} \mathrm{~d}^{-1} ; \mathrm{n} / \mathrm{N}$ é a razão da insolação média mensal.

Usando dados obtidos nos lisímetros de Davis, no estado da Califórnia (clima semiárido), com gramado, Hargreaves e Samani (1985) propuseram um novo método para a estimativa da evapotranspiração (PEREIRA et al., 1997). O método proposto por Hargreaves e Samani (1985) para a estimativa da evapotranspiração de referência é uma alternativa para situações em que não se têm dados medidos de radiação solar à superfície, umidade relativa e velocidade do vento:

$$
\mathrm{ET}_{\mathrm{oHS}}=0,0023\left(T_{x}-T_{n}\right)^{0,5}\left(T_{m}+17,8\right) R_{a} \cdot 0,408
$$

Em que:

$\mathrm{T}_{\mathrm{x}}$ - temperatura máxima do ar, ${ }^{\circ} \mathrm{C}$;

$\mathrm{T}_{\mathrm{n}}$-temperatura mínima do ar, ${ }^{\circ} \mathrm{C}$;

$\mathrm{T}_{\mathrm{m}}$-temperatura média do ar, ${ }^{\circ} \mathrm{C}$;

$\mathrm{R}_{\mathrm{a}}$-radiação solar no topo da atmosfera, MJ m ${ }^{-2} \mathrm{~d}^{-1}$;

\section{RESULTADOS E DISCUSSÕES}

A Tabela 1 apresenta as médias mensais da ETo determinadas através dos métodos de Penman-Monteith FAO, Hargreaves-Samani, Radiação e Blaney-Cridle 


\section{ESTIMATIVA DA EVAPOTRANSPIRAÇÃO POR DIFERENTES MÉTODOS PARA A CIDADE DE QUIXERAMOBIM, CEARÁ}

Tabela 1 - Valores médios mensais de evapotranspiração dos modelos Penman-Monteith FAO (Eto_PM), Hargreaves-Samani (HS), Radiação (RAD) e Blaney-Cridle utilizados para a cidade de Quixeramobim-CE.

\begin{tabular}{ccccc}
\hline Mês & Eto_PM & HS & RAD & Blaney-Criddle \\
\hline jan & 6,31 & 5,70 & 6,65 & 4,76 \\
fev & 5,37 & 5,64 & 5,53 & 3,89 \\
mar & 4,45 & 5,13 & 4,67 & 3,21 \\
abr & 4,57 & 4,88 & 5,00 & 3,52 \\
mai & 5,15 & 4,83 & 5,44 & 4,44 \\
jun & 4,78 & 4,63 & 5,01 & 4,30 \\
jul & 4,40 & 4,43 & 4,47 & 3,75 \\
ago & 5,94 & 5,18 & 6,59 & 5,14 \\
set & 6,40 & 5,73 & 6,81 & 4,93 \\
out & 6,85 & 6,13 & 7,29 & 5,13 \\
nov & 6,70 & 5,83 & 7,33 & 5,22 \\
dez & 6,72 & 5,84 & 6,91 & 5,01 \\
\hline Média & $\mathbf{5 , 6 4}$ & 5,33 & 5,97 & 4,44 \\
\hline
\end{tabular}

O método de Blaney-Criddle subestimou os valores de ETo quando se comparado ao método padrão adotado de Penman-Monteith (Figura 2). Já o método da Radiação superestimou os valores, apresentando tendências maiores em relação ao método adotado como padrão durante todo o ano. O método de Hargreaves-Samani apresentou períodos de tendências maiores no período chuvoso (fev-mai), e tendências menores no período seco (jun-dez), suavizando o comportamento.

Tendências similares para o métodos de Hargreaves-Samani foram observadas por Palaretti et al. (2014) avaliando as regiões citrícolas em São Paulo. Esta tendência é um reflexo da variação espacial da temperatura média do ar mensal nas diferentes regiões do Estado. (GOMES et al., 2005).

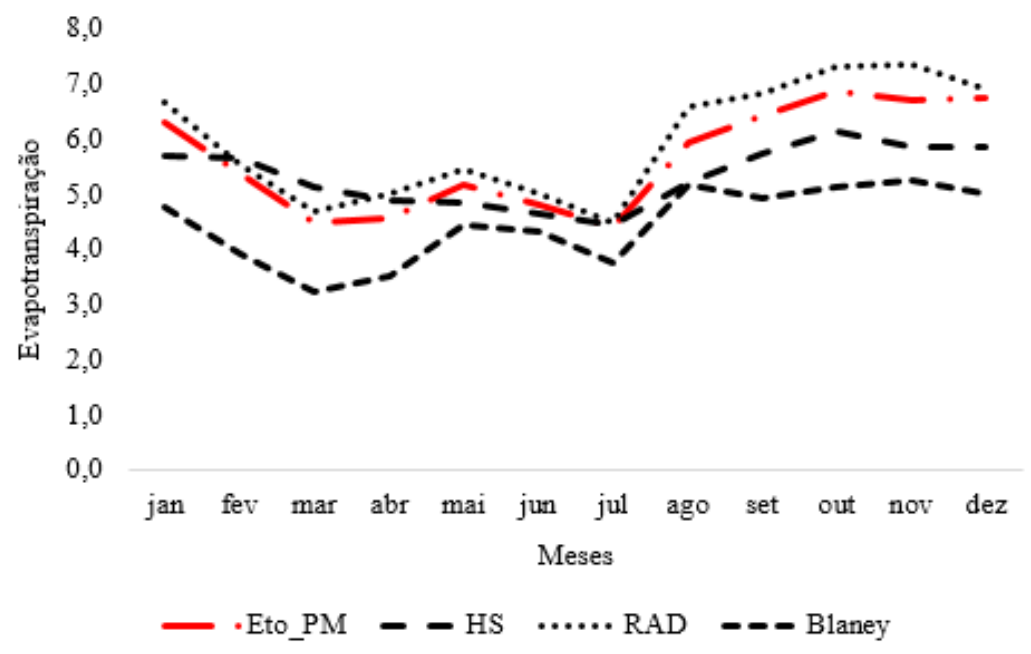

Figura 2 - Variação dos valores estimados de ETo de cada modelo analisado para a cidade de QuixeramobimCE 
Observa-se na figura 3 que o método que melhor se ajustou ao adotado como padrão foi o método da Radiação, gerando um coeficiente de determinação $\left(\mathrm{r}^{2}\right)$ de 0,97 , indicando uma excelente correlação. Tagliaferre et al. (2010) compararam diferentes métodos de estimativa de evapotranspiração de referência para a região de Eunápolis-BA e verificaram que o método da Radiação apresentou melhores desempenhos para as estimativas de ETo em todos os períodos de tempo estudados. Estes autores observaram também que o método de Hargreaves-Samani não foi satisfatório para a estimativa da evapotranspiração
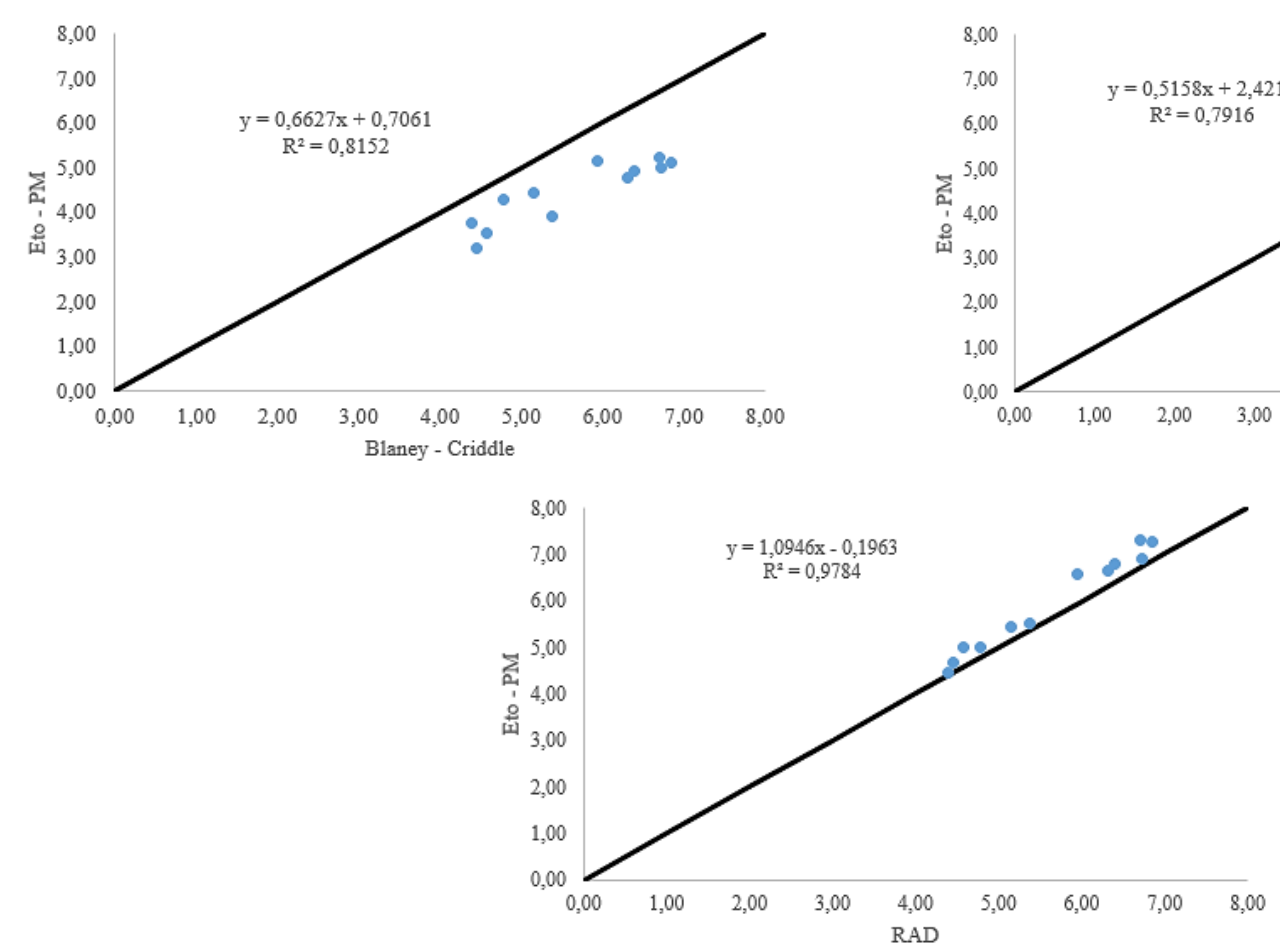

Figura 3 - Correlação entre os valores de ETo - PM e os métodos de Blaney-Criddle, Hargreaves-Samani e Radiação.

\section{CONCLUSÕES}

Quando se tem escassez de dados, o método de Radiação é o mais indicado tendo em vista o menor número de entradas e o maior coeficiente de determinação com o método padrão de PenmanMonteith.

O método de Hargreaves-Samani tem a característica de suavização da evapotranspiração, fato que pode ocasionar

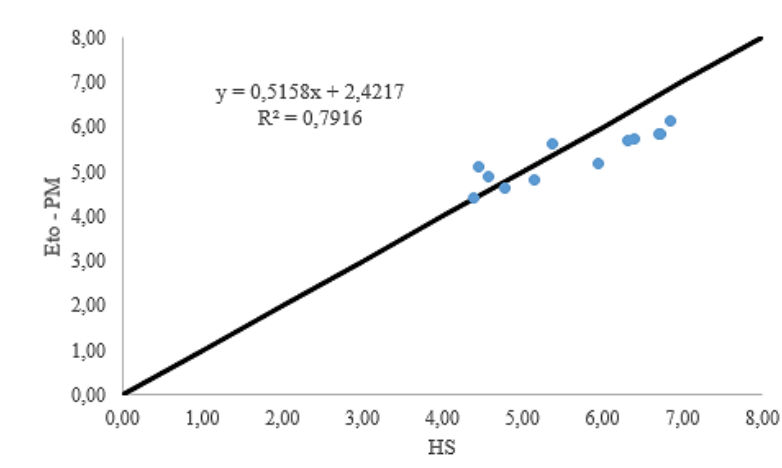

Para Carvalho et al. (2011) quando há ausência de dados climáticos ocorre a impossibilidade da aplicação do método adotado como padrão pela FAO 56 (Penman-Monteith), porém pode ser usado o método que melhor se ajuste as condições climáticas da região que requeira menos dados de entrada.

$\mathrm{O}$ métodos de Blaney-Criddle e Hargreaves-Samani apresentaram um $\mathrm{r}^{2}$ de 0,81 e 0,79, respectivamente, apresentando um correlação baixa. Alencar et al. (2011) constatou também uma baixa relação para HargreavesSamani estudando a evapotranspiração em Minas Gerais.

erros quando aplicado estes valores para cálculos de irrigação.

\section{REFERÊNCIAS}

ALENCAR, L. P.; SEDIYAMA, G. C. ; WANDERLEY, H. S. ; ALMEIDA, T. S. ; DELGADO, R. C. Avaliação de Métodos de Estimativa da Evapotranspiração de Referência para Três Localidades no Norte de Minas 
Gerais. Engenharia na Agricultura (Impresso), v. 19, p. 437-449, 2011.

ALLEN, R. G.; PEREIRA, L. S.; RAES, D.; SMITH, M. Crop evapotranspiration Guidelines for computing crop water requirements. Roma: FAO, 1998. 300p. (FAO Irrigation and Drainage Paper, 56).

ANDRADE, E. M.; RODRIGUES, M. M. A.; MENDONÇA, M. A. B.; CHAVES, L. C. G.; FEITOZA, R. M. Investigation of the maximum and minimum temperatures in the semi-arid region of northeastern Brazil. Revista Agro@mbiente On-line, v. 5, n. 2, p. 82-87, 2011.

BLANEY, H. F.; CRIDDLE, W. D. 1950. Determining Water Requirements in Irrigated Area from Climatological Irrigation Data, US Department of Agriculture, Soil Conservation Service, Tech. Pap. No. 96, $48 \mathrm{p}$.

CARVALHO, L. G.; RIOS, G. F. A.; MIRANDA, W. L.; CASTRO NETO, P. C. Evapotranspiração de referência: uma abordagem atual de diferentes métodos de estimativa. Pesquisa Agropecuária Tropical, v. 41, n. 3, p. 456-465, 2011.

DOORENBOS, J, AND W. O. PRUITT. 1977. Guldel Ines for predicting Crop Water Requirements. FAO Irrigation and Drainage paper 24, Food and Agriculture Organization of the United Nations, Rome, Italy, 144 p.

DOORENBOS, J.; KASSAM, A.H. Efeito da água no rendimento das culturas. Campina Grande: UFPB, 1994. 306p. (Estudos FAO: Irrigação e Drenagem, 33).

ESTEVES, B. S.; MENDONÇA, J. C.; SOUSA, E. F.; BERNARDO, S. Avaliação do Kt para estimativa da evapotranspiração de referência (ETo) em Campos dos Goytacazes, RJ. Revista Brasileira de Engenharia Agrícola e Ambiental, v. 14, n. 3, p. 274-278, 2010.
GOMES, A. A. N.; ANDRADE JÚNIOR, A. S.; MEDEIROS, R. M. Evapotranspiração de referência mensal para o Estado do Piauí. Revista Brasileira de Engenharia Agrícola e Ambiental, Campina Grande, v. 9, n. 4, p. 560-564, 2005.

HARGREAVES, G.H., SAMANI, Z.A. 1985. Reference crop evapotranspiration from temperature. Transaction of ASAE, v. 1, n. 2, p. 96-99.

JABLOUN, M.; SAHLI, A. Evaluation of FAO56 methodology for estimating reference evapotranspiration using limited climatic data application to Tunisia. Agricultural Water Management, v. 95, n. 6, p. 707-715, 2008.

MINUZZI, R. B.; RIBEIRO, A. J.; SILVA, D. O.; KUNESKI, A. C. Estimativa da evapotranspiração de referência diária por Penman-Monteith FAO com dados de temperatura do ar para Santa Catarina. Irriga, v. 19, n. 3, p. 548-558, 2014.

PALARETTI, L. F.; MANTOVANI, E. C.; SEDIYAMA, G. C. Comparação entre métodos de estimativa da evapotranspiração de referência (ETo) em regiões citrícolas paulistas. Engenharia Agrícola, v. 34, n. 1, p. 38-47, 2014.

PEREIRA, A. R.; VILLA NOVA, N. A.; SEDYIAMA, G. C. Evapo(transpi)ração. Piracicaba: FEALQ, 1997. 183 p.

D'ANGIOLELLA G., VASCONCELLOS, V. L. D., ROSA, J. W. C. Estimativa e espacialização do balanço hídrico na mesorregião sul da Bahia. In: XII Simpósio Brasileiro de Sensoriamento Remoto. Anais... Goiânia, Brasil, p. 83-90, 2005.

SENTELHAS, P. C.; GILLESPIE, T. J.; SANTOS, E. A. Evaluation of FAO PenmanMonteith and alternative methods for estimating 
reference evapotranspiration with missing data in Southern Ontario, Canada. Agricultural Water Management, v. 97, n. 05, p. 635-644, 2010.

TAGLIAFERRE，C.; SILVA， R.; ROCHA, F.; SANTOS, L. Estudo comparativo de diferentes metodologias para determinação da evapotranspiraçao de referência em
Eunapolis - BA. Revista Caatinga, v. 23, n. 1, p. 103-111, 2010.

TRAJKOVIĆ, $\quad$ S.; $\quad$ STOJNIĆ, $\quad$ V.; GOCIĆ, M. Minimum weather data requirements for estimating reference evapotranspiration. Architecture and Civil Engineering, v. 9, n. 2, p. 335-345, 2011. 